\title{
Hybrid WIMAX and DVB-H Emulator for Scalable Multiple Descriptions Video Coding Testing
}

\author{
C. H. Liew and S. Worrall \\ Centre for Communication Systems Research \\ University of Surrey \\ Guildford, Surrey, UK \\ \{c.liew, s.worrall\}@ surrey.ac.uk
}

\author{
M. A. Mota and A. Navarro \\ Telecommunications Institute \\ University of Aveiro \\ Aveiro, Portugal \\ $\{$ mmota, navarro\}@av.it.pt
}

\begin{abstract}
This paper discusses the construction of a wireless emulator for the study of $\mathbf{H . 2 6 4}$ Scalable Video Coding (SVC) and $\mathbf{H . 2 6 4}$ based Scalable Multiple Description Coding (SMDC) transmission. This emulator is constructed as a part of European SUIT project for examining the benefit of SVC and SMDC for digital TV broadcasting. The individual component of the emulator and the technique to generate error pattern files will be discussed in this paper. An example of the emulator usage is finally shown.
\end{abstract}

\section{Keywords - WIMAX, DVB, Wireless, Emulator}

\section{INTRODUCTION}

HIS paper discusses a Hybrid WIMAX and DVB-H TEmulator (HIWIDEM) for the testing of H.264 Scalable Video Coding (SVC) [1][2] and Scalable Multiple Descriptions Coding (SMDC) streams over WIMAX and DVB wireless bearer for European Scalable Ultra Fast Interactive TV (SUIT) project [3]. SVC is very suitable for fast and dynamic adaptation of video source bit rate. SVC utilizes concept of temporal scalability, spatial scalability and SNR scalability which respectively provide ease for frame rate adaptation, video resolution adaptation and video quality adaptation to adjust the video source in accordance to device capability and channel bandwidth. Multiple Description Coding (MDC) [4] provides extra error resilience against channel error by generating multiple streams (descriptions) of equal importance from a single video stream. Individual stream can be transmitted over different channel and decoded for independent viewing. However, if multiple streams are received simultaneously, the decoded quality is enhanced. Within the SUIT project, we endeavor to facilitate best of the both SVC and MDC to guarantee high quality digital video broadcasting by combining both schemes into a highly
Scalable MDC (SMDC) where each stream (description) is easily adaptable. The SUIT project will eventually prototype an end-to-end solution of broadcasting comprising encoder, playout with dynamic resource management, wireless transmission and decoder. However, it is important to examine SVC and SMDC in advance of the field trial in order gauge an estimation of performance in terms of video quality. This will give an indication of achievable performance allowing the estimation of equipment requirements and actual transmission requirements e.g. power budget and bit error rate acceptable. For this purpose, we have constructed a Linux-based wireless emulator called HIWIDEM consisting of WiMAX and DVB-H bearers for realistic testing of SVC and SMDC. The HIWIDEM uses two stage processes: pre- simulation of error pattern files and real time packet processing using packet filtering capability of Linux system. The following sections will discuss HIWIDEM in detail. The organization of the paper is as follows. Section II introduces the simplified SUIT architecture. Based on this basic model, the HIWIDEM is introduced in Section III. The inner working of HIWIDEM will be explained. Section IV presents procedures to generate error pattern traces. The simulated trace will be used in the HIWIDEM emulator. Finally, Section V presents the resulting emulator and example usage.

\section{SUIT ARCHITECTURE}

This paper does not intend to describe the complete architecture of SUIT system. The complete system will be described in separate paper in this proceeding [5]. This section will only present a simplified implementation of SUIT architecture and discusses the emulator accordingly. In Figure 1, two SMDC descriptions are synchronously streamed from server 


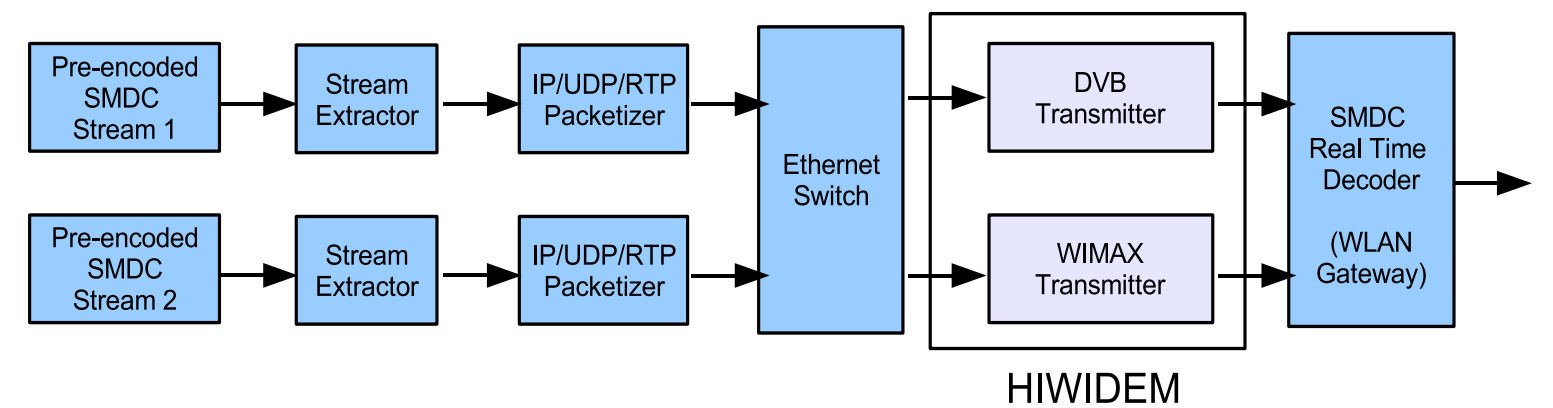

Figure 1: Simplified implementation of SUIT architecture

to DVB and WIMAX channel. Video files are preencoded using SMDC codec and stored on the streaming server. In the server, a stream extractor will parse and extract SMDC descriptions or the NALUs (i.e. H.264 Network Abstraction Layer Units) [2] from the pre-encoded video files. The NALUs encoded using SMDC are scalable and can be truncated easily for source bit rate adaptation. In particular, the SNR Scalably (i.e. Fine-Grained Scalability) coded NALUs can be truncated at any point of bitstream. NALUs are then packetized into IP/UDP/RTP packets according to RFC3984 [6] and transmitted into the Ethernet in multicast mode. In the simplified prototyped in Figure 1 , both descriptions are streamed from a single video server, but routed to WIMAX and DVB-H transmitters with an Ethernet Switch. A SUIT receiver (i.e. WLAN Gateway) received and combined both SMDC streams from WIMAX and DVB-H back into a single H.264 AVC stream for transmission around the residence or public hotspot. Because WIMAX and DVB-H uses different frequency band, this creates frequency diversity and is exploited by SMDC for robust digital TV transmission. Signal fade is unlikely to happen simultaneously since WIMAX and DVB-H uses different frequency band that are uncorrelated. Thus the receiver will receive at least one stream with high probability. As long as either one description is received, the receiver will be able to view the transmitted program (if both description is received, the video quality is enhanced). For the first stage of testing, we have considered using an emulator called HIWIDEM in Figure 1 for controlled environment lab testing before the actual field trials. HIWIDEM models the behavior of wireless channel and offers the flexibility of instantaneous parameters tuning (e.g. speed, Signal to Noise Ratio (SNR), and Modulation and Coding Scheme (MCS)). HIWIDEM offers a cheap and fast way to test SVC and SMDC in a more controlled way. HIWIDEM is illustrated in detail in the next section.

\section{HIWIDEM}

HIWIDEM is divided into a two stage processes for channel emulation: pre-simulation of error pattern files and real time packet processing stage (using error pattern files). The pre-simulated error patterns are consist of traces for different Signal to Noise Ratio (SNR) for different Modulation and Coding Scheme (MCS) generated using physical layer WIMAX and DVB-H baseband simulator. In summary, HIWIDEM captures incoming packet, process the packet, and decide whether to drop or to a release the packet back into the network according to the channel error behavior. HIWIDEM is situated in a Linux-based PC with four Ethernet cards as portrayed in Figure 1. Each pair of Ethernet card is linked together as an Ethernet bridge for emulation of WIMAX and DVB bearer respectively. The Ethernet bridge functionality is supported natively in Linux-base operating system with kernel version larger than 2.6.x. An open source scripts called bridge-utils [7] is used for setting up the bridge and linking of Ethernet interfaces.

The functions of the HIWIDEM are shown in Figure 2. The emulator performs filtering on the incoming packets on the Ethernet Bridge. The packet filtering module utilizes iptable and netfilter software [8], also supported natively in Linux system, to capture packets streamed to different IP multicast address. The packet filtering module examines the destination IP address 


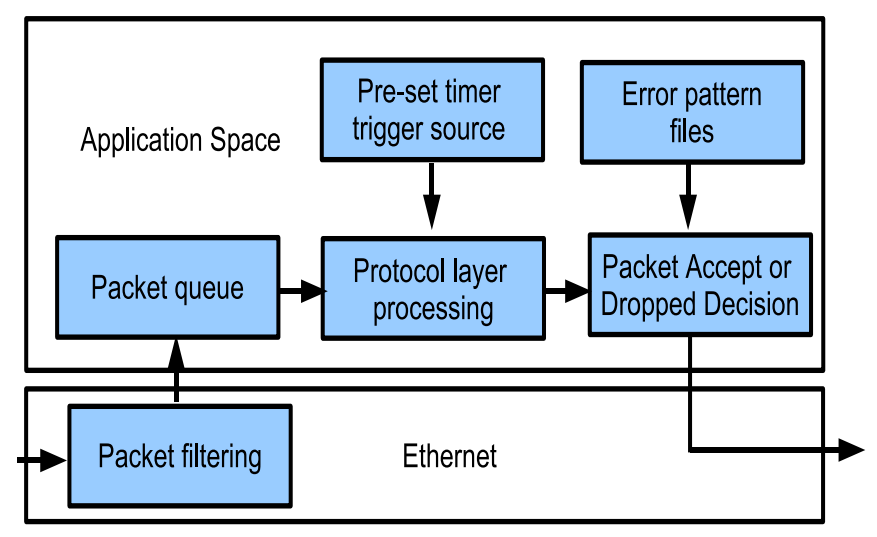

Figure 2: Inner functions of HIWIDEM

of packet and passes it to separate WIMAX and DVB$\mathrm{H}$ queue for transmission. The packet filtering module is programmed using multi-threading API to process the UDP sockets regularly. WIMAX and DVB-H protocol layer processing engine will process the queue regularly. A pre-set timer fires off periodically to trigger the processing engine, e.g. $5 \mathrm{~ms}$ for WIMAX TDD Frame.

WIMAX and DVB processing engine perform underlying protocol layer functions such as packet fragmentation, MAC encapsulation etc, according to the WIMAX [9] and DVB-H [10] standard. For example, WIMAX and DVB-H protocol layer processing are shown in Figure 3 and Figure 4. Dummy protocol headers are inserted to construct smaller source blocks for transmission. At this stage, the source blocks are compared to pre-simulated error pattern files to decide if the transmission is successful. If the transmissions are not successful, the packet that is encapsulated in the source blocks will be dropped or released back into the network otherwise. The presimulated error patterns are composed of traces of different SNRs for different MCSs generated using physical layer WIMAX and DVB baseband simulator.

\section{TRACE GENERATION PROCEDURES}

This section discusses the error pattern generation procedures for both WIMAX and DVB-H. For both cases, simplification have been made in order to reduce the overhead and disk storage. This will allow fast execution of emulator without delay, i.e. pseudoreal time, performing queue processing according to the pre-set timer, e.g. $5 \mathrm{~ms}$ for WIMAX.

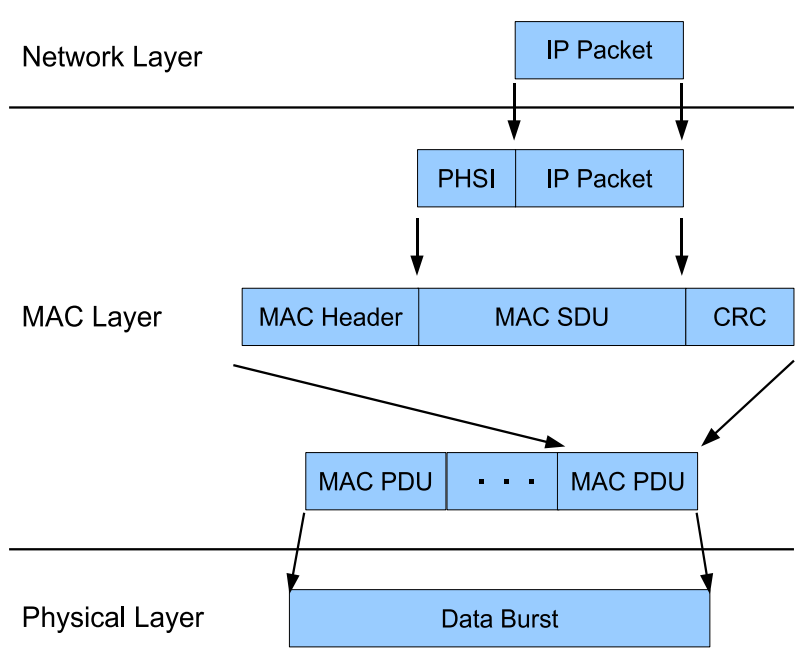

Figure 3: WIMAX protocol layer processing

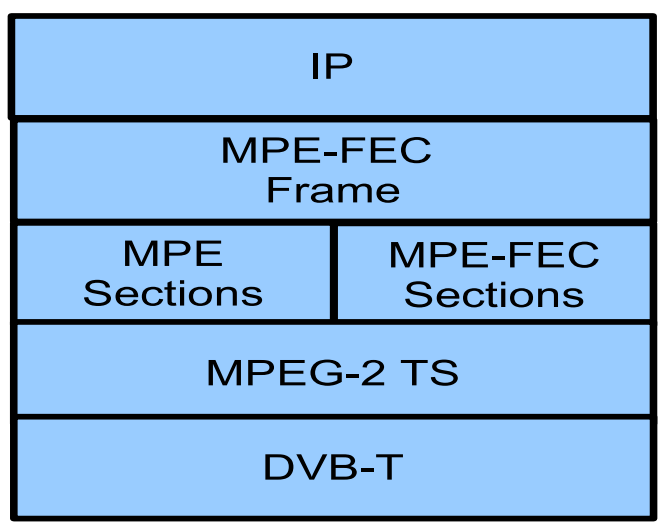

Figure 4: DVB-H protocol layer processing

Figure 5 illustrates the WIMAX Time Division Duplex (TDD) physical layer transmission frame [9]. During the error pattern generation, we have considered only downlink (DL) sub-frame. Basically the DL Data Burst $\# \mathrm{x}$ region is the allocated region for data transmission. A data burst, as shown in the figure, allows the user to transmit data depending on the MAC scheduler allocation decision. Essentially the DL sub-frame is composed of a number of slots. For our case, the DL sub-frame is composed of 30 subchannels x 13 time symbols $=390$ slots. Each slot comprises 48 QAM symbols which allow a certain number of data bits depending on the Modulation and Coding Scheme (MCS) of the system. 


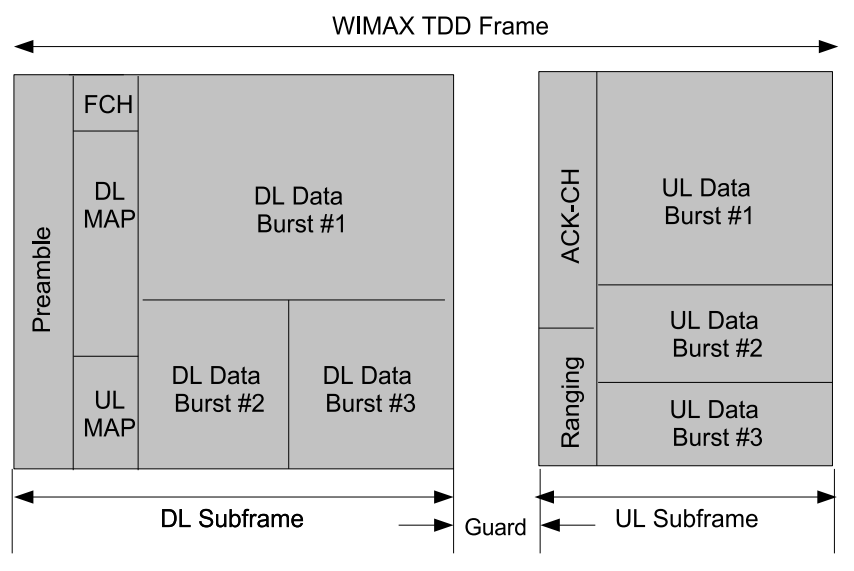

Figure 5: WIMAX TDD frame

In order to reduce data storage requirements, the error pattern files are saved in the form of a data slot error pattern instead of a bit error pattern. The data slot error patterns are obtained by comparing all the data bits within original data slot to the transmitted data slot. If there is any bit error within the data slot, it is then declared as an error. Note that we have not specifically assumed any IP packet size or data burst size within a physical layer frame. Also, no MAC layer packet encapsulation procedure is performed. This decision was made to allow flexibility on the choice of packet size and the data throughput during video transmission simulation. Data slots can be aggregated at later stage to different burst sizes for different data throughput. The user of the error pattern trace will need to perform the procedures shown in Figure 3. The error pattern files are generated with Partial Usage SubChannel (PUSC) Scheme, Duo-binary Convolutional Turbo Coding (CTC), and MCS of QPSK-1/2, QPSK-3/4, 16QAM-1/2, 16QAM-3/4, 64QAM-1/2. Maximum FEC code block size has been assumed for the trace generation. The maximum code block size is MCS mode dependent, as defined in the IEEE 802.16e-2005 standard. OFDMA has been considered with 1024 FFT size. The mobile speed is $60 \mathrm{kmph}$.

As for DVB-H, the MPE-FEC transmission frame [10] is shown in Figure 6. It consists of two sections: Application Data Table (ADT) and Reed Solomon Data Table (RSDT). ADT is used to carry data from a video service while RSDT is used to carry the Reed Solomon parity bytes for enhanced error resilience in mobile environment. ADT has fixed number of columns, i.e. 191, while the number of rows is variable to accommodate different service bit rate requirements. The maximum number of rows is 1024

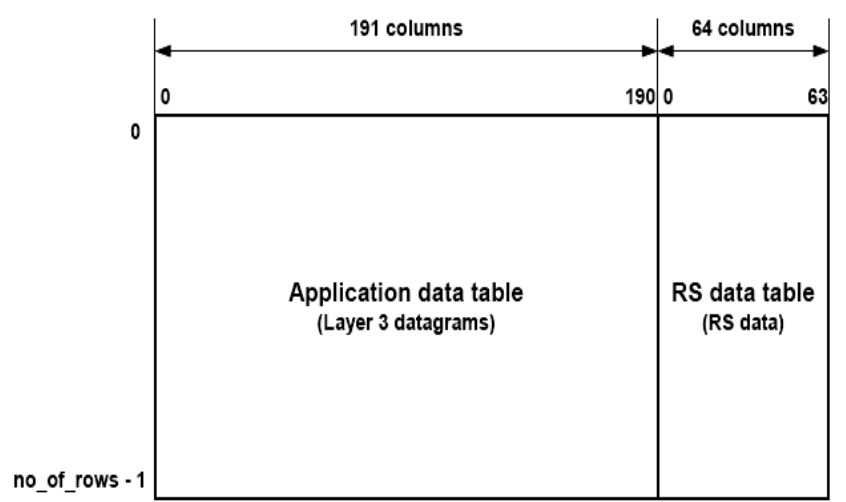

Figure 6: DVB-H transmission frame

which capped the maximum burst size to $(191+64) \mathrm{x}$ 1024 × $8=\sim 2$ Mbit for each MPE-FEC frame. Each MPE-FEC frame is then fragmented and encapsulated into MPEG-2 Transport Stream (TS) format before mapping it into DVB-T physical layer frame.

Similar to WIMAX, simplification has been assumed to avoid overhead. This avoids the need to process error pattern files at bit level which consumes more data storage and computational resources for protocol layer processing. We have assumed number of rows of 512 and the IP packet size of 512 bytes. Thus each packet fits exactly into the each column of MPE-FEC frame. The packet error pattern generate by comparing transmitted packet to the received packet. The packet is declared as error if the received MPE-FEC column that carries the packet consists of error bytes. The error pattern files have been generated using 64QAM-3/4, FFT size of 2048, speed of $60 \mathrm{kmph}$ and $140 \mathrm{kmph}$.

\section{RESULTS AND DISCUSSIONS}

Figure 7 shows the resulting HIWIDEM wireless emulator for testing of SUIT architecture. HIWIDEM models the behavior of wireless channel and offers the flexibility parameters tuning. The Graphical User Interface (GUI) was programmed using cross platform QT Trolltech GUI C++ Toolkit [11]. We have used the open source license free version for Linux platform. The tunable parameters of HIWIDEM are Signal to Noise Ratio (SNR), Modulation and Coding Scheme (MCS) and it can easily be configured to capture packets of different SVC or SMDC streams by setting the destination address of a stream. HIWIDEM offers a cheap and fast way to test SVC and SMDC in a 


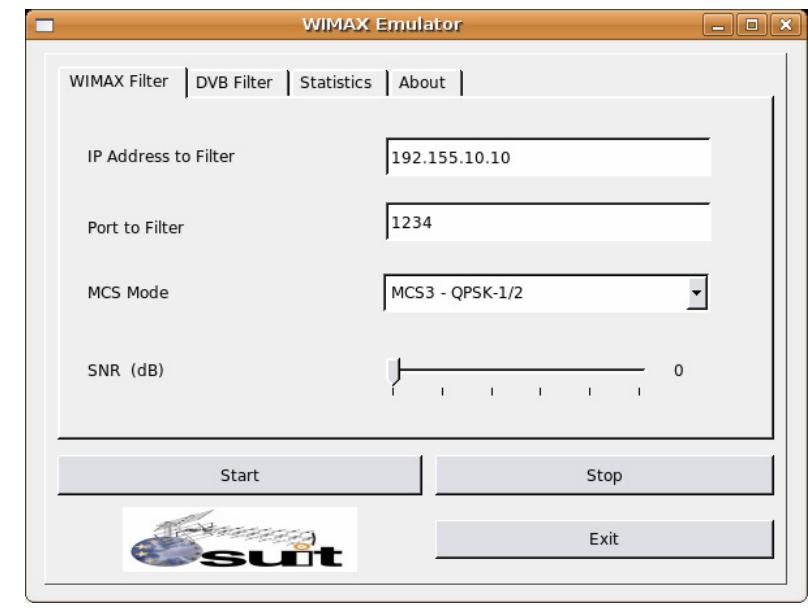

Figure 7: Hybrid WIMAX and DVB-H Emulator

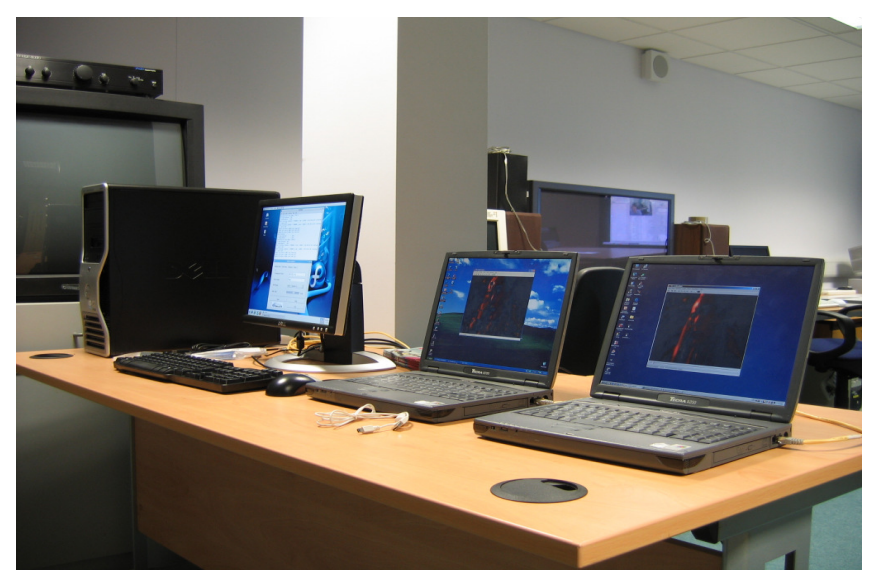

Figure 8: Usage Example for Video Testing

controlled environment to allow simple debugging of algorithms. This allows easy testing of video to give early indication of quality and enables the adjustment and optimization of system and video codec. HIWIDEM has been successfully integrated into simplified SUIT architecture shown in Figure 1. However, we only present a usage example for illustrative purposes. Figure 8 shows the example setup testing platform. The laptop on the middle is used as the streamer while the laptop on the right is used as the receiving client. The PC on the left is configured and installed with HIWIDEM software to perform wireless channel emulation. VideoLAN VLC [12] is used as the streamer and also the receiving client. Figure 9 and Figure 10 shows the video "Lord of the Ring" transmitted to the WIMAX channel and video "Lord of the Ring" received. The SNR value of

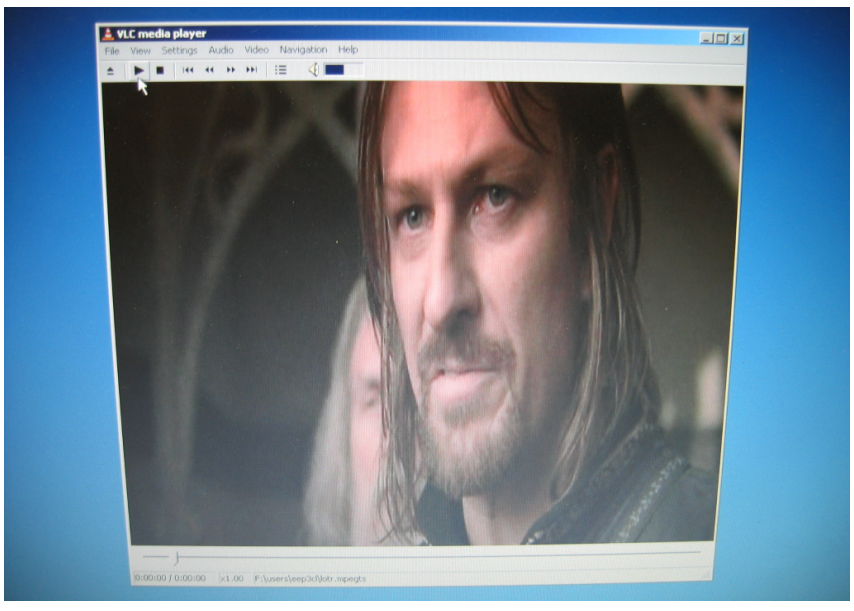

Figure 9: VLC Streamed Video "Lord of the Ring"

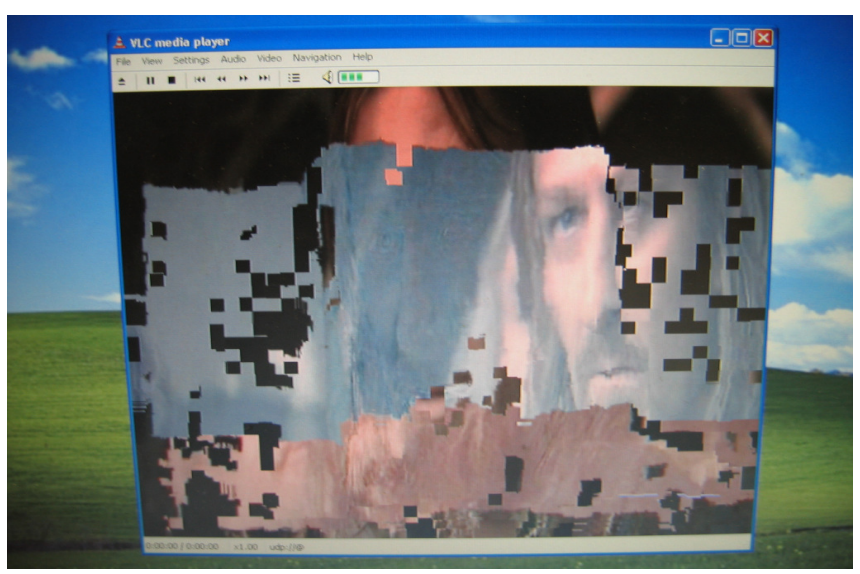

Figure 10: VLC Received Video "Lord of the Ring"

was set to relatively low value. As seen in Figure 10, the quality of video degrades significantly due to packet drops as a result of poor channel conditions. Objective quality measurement using Peak Signal to Noise Ratio (PSNR) can be performed easily by comparing transmitted and the received stream.

This paper has discussed an emulator called HIWIDEM for the testing of SVC and SMDC video transmission over WIMAX and DVB channel. The basic construct of Linux-based emulator has been presented. The technique for pre-simulated trace was also discussed in view of reducing the data storage and computational requirement. Finally the resulting emulator and usage example of the HIWIDEM were illustrated. HIWIDEM, will be used in the project later to generate preliminary quality estimation of SVC and SMDC encoded video before the actual field trials. 


\section{ACKNOWLEDGEMENT}

The authors would like to acknowledge the funding of European Commission (EC) for the Scalable Ultra-fast interactive Interoperable Television (SUIT) project in which this work was performed within.

\section{REFERENCES}

[1] T. Wiegand, G. J. Sullivan, G. Bjontegaard and A. Luthra, "Overview of the H.264 / AVC Video Coding Standard", IEEE Transactions on Circuit and Systems for Video Technology, vol 13, issue 7, July 2003

[2] H. Schwarx, D. Marpe and T. Wiegand, "Overview of the Scalable H.264 / MPEG4-AVC Extension" , Proc. IEEE International Conference on Image Processing, Atlanta, GA, USA, Oct 2006

[3] EU IST FP6 Project: "Scalable, Ultra-fast and Interoperable Interactive Television (SUIT)", http://suit.av.it.pt

[4] W. Yao, A. R. Reibman and Shunan Lin, "Multiple Description Coding for Video Delivery", Proceedings of the IEEE, vol 93, issue 1, Jan 2005 pages: 57- 70

[5] A. Navarro, "SUIT - Scalable, Ultra-fast and Interoperable Interactive Television," ISCE 2007, accepted

[6] S. Wenger, M. M. Hannuksela, T. Stockhammer, M. Westerlund, D. Singer, "RTP Payload Format for H.264 Video", RFC3984, Feb 2007

[7] Linux Bridge Utilities, http://linuxnet.osdl.org/index.php/Bridge

[8] Netfilter Project, http://www.netfilter.org

[9] "Part 16: Air Interface for Fixed and Mobile Broadband Wireless Access Systems. Amendments 2: Physical and Medium Access Control Layers for Combined Fixed and Mobile Operation in Licensed Bands and Corrigendum 1," Dec 2005

[10]ETSI EN $302 \quad 304$, v.1.1.1, "Digital Video Broadcasting: Transmission System for Handheld Terminals", ETSI standard, November 2004.

[11]QT Trolltech GUI Programming Toolkit, http://trolltech.com/products/qt

[12] VideoLAN VLC, http://www.videolan.org/vlc/ 